\title{
Effects of Clustering and Successive Paging on Reporting Center Scheme: A Cost Analysis
}

\author{
Vijendra S. Bhadauria \\ Computer Science \& Engg. \\ S.I.R.T.E. \\ Bhopal (M.P.) India
}

\author{
Ravindra Patel \\ Computer Applications \\ U.I.T., R.G.P.V. \\ Bhopal (M.P.) India
}

\author{
Sanjeev Sharma \\ School of I.T., \\ U.I.T., R.G.P.V. \\ Bhopal (M.P.) India
}

\begin{abstract}
One of the key issues in cellular mobile communication is to find current location of mobile terminals to deliver the service, which is known as location management. Although dynamic location management schemes show better performance but static schemes are more popular due to their simplicity. One such static scheme is reporting center in which some cells are designated as reporting centers. This paper proposes a basic criteria for performing clustering and successive paging in reporting center scheme and also analyzes the cost effectiveness of three different versions of reporting center scheme: Simple, Clustered, and Combination of clustering with successive paging. Simulation results show that the combination of clustering with successive paging outperforms the other two versions independent of call to mobility ratio.
\end{abstract}

\section{General Terms}

Location management.

\section{Keywords}

Cellular mobile network, reporting center, location management, registration, paging, clustering.

\section{INTRODUCTION}

Cellular mobile communication enables mobile terminals (MTs) to communicate with each other independent of their location. The network is divided into cells and each cell is served by a base station (BS) that communicates with MTs in its cell over pre-assigned radio frequencies. Groups of several cells are connected to a mobile switching center (MSC) through which, calls are routed to telephone networks. It interfaces between mobile phones (via BS) and the public switched telephone network (PSTN). Home Location Register (HLR) and the Visitor Location Register (VLR) are the databases used for LM in mobile networks. Location management (LM) involves tracking of MT's up-to-date location, which moves freely across different cells in order to provide it services. Two basic operations in LM are: Location Update (LU) and Paging [1-4]. Costs of these two operations are negatively correlated so it is very difficult but necessary to provide good tradeoff between them. Basically LM schemes can be classified into static and dynamic. Under static schemes, every MT undergoes same number of updates when passes through a specific region. One such scheme is reporting center in which, some of the cells are designated as reporting centers and all nearby cells up to the next reporting center belong to vicinity of same reporting center. MT updates its location whenever it crosses vicinity of its current reporting center, which happens only when it enters into another reporting center and therefore a LU is triggered. To deliver a call, network pages current reporting center and its whole vicinity simultaneously to locate the target MT.

The reporting cells form a solid line of barrier, which means a user will have to enter one of the reporting cells to get to the other side [1]. For example, in Fig.1 (a), cells 1, 5, 8, 9, 10, 11 and 15 are reporting centers. Vicinity of reporting center 1 consists of cells $0,2,3,4,6,7$ and 1 itself. Similarly, vicinity of reporting center 9 consists of cells $0,4,12,13,14$ and 9 . An MT in cell 13 must cross a reporting center to enter into cell 6 and therefore LU will be triggered.

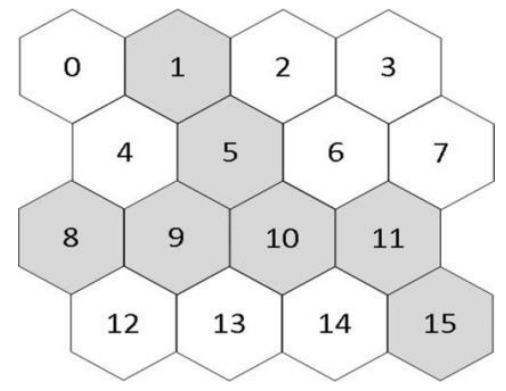

(a) Bounded non-reporting cell configuration

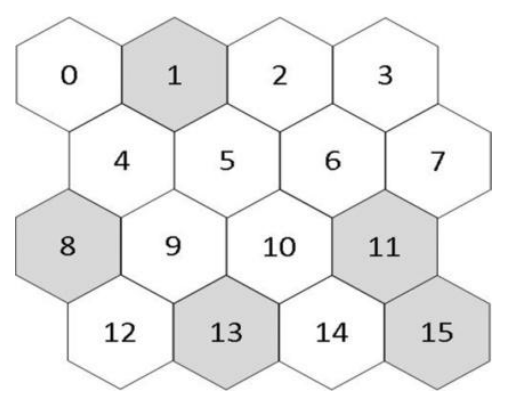

(b) Unbounded non-reporting cell configuration

Fig 1: Configurations of reporting center scheme (gray cells are reporting centers)

A cell may fall under vicinity of more than one reporting centers. For example in Fig.1 (a), cells 2, 3, 6 and 7 fall under the vicinity of reporting centers $1,5,10$ and 11 . That means whether the reporting center of an MT is either cell 1, 5, 10 or 11 , these four cells will always be paged whenever a call arrives of that MT. Under reporting center scheme, non reporting cells may be bounded or unbounded. Fig.1 (a) and (b) shows bounded and unbounded non reporting cell configurations respectively. 
Next section gives an overview of LM cost function, Section3 and 4 presents the proposed clustering technique and successive paging scheme respectively, Simulation results and performance comparison is done in section-5, and section-6 concludes the paper.

\section{LOCATION MANAGEMENT COST}

A number of LM schemes have been proposed by various researchers so there must be some framework that can be used to compare one scheme with the other. LM cost function comprises of two main components: updating cost and paging cost. Updating cost is the cost due to location updates performed by MTs in the network whereas paging cost is caused by the network during location inquiries while locating an MT [5]. There are some other parameters that influence the total LM cost [6] such as cost of database management in LU operation, cost in terms of wired line (backbone) network bandwidth used (that connects base stations to each other). Mostly these costs are assumed to be constant for all LM schemes. So combination of LU and paging cost are considered to be sufficient to compare different LM schemes [1], [6-10].

$$
\text { Total LM Cost }=(\mathrm{C} \times \mathrm{NLU})+(\mathrm{NP})
$$

Where NLU denotes the number of location updates performed during simulation time $\mathrm{T}$, NP denotes the number of paging operations performed during time $\mathrm{T}$ and $\mathrm{C}$ is a constant, which is the ratio of single LU cost to the cost of paging single cell. It is believed that the cost of a $\mathrm{LU}$ is ten times the cost of a paging operation [6], [11], [12]. In light of this fact, we used $\mathrm{C}=10$.

Reporting center scheme decreases LU cost but increases the paging cost [13]. In unbounded non-reporting cell configuration, since the vicinity of reporting centers are not bounded therefore paging cost may increase noticeably as compared to bounded non-reporting cell configuration. This paper presents a simple criterion for clustering reporting centers in bounded non reporting cell configuration that decreases total LM cost but since clustering always increases the paging cost therefore a successive paging scheme is proposed which, when combined with clustering technique, curtails both updating cost and paging cost resulting into noteworthy reduction in total LM cost.

\section{CLUSTERING CRITERION}

As shown in Fig.2, cells 1, 6, 8, 9, 10, 11, 14 and 17 are reporting centers. If an MT moves between two adjacent reporting centers then it updates the location each time it leaves current reporting center and enters into the other. Such situation can be avoided by clustering those adjacent reporting centers. For example, if cell 9 and 10 are clustered then MT does not need to update its location while moving between these two reporting cells but since the vicinity of clustered reporting centers will include the vicinity of all clustered members [13] therefore after clustering, vicinity of this cluster will include cells $0,2,3,4,5,6,7,9,10,12,13,15,16,18$ and 19. This will reduce the number of location updates because now the MT will not trigger an LU while moving between cell 9 and 10 but it will double the paging cost for MTs present in this cluster as the number of cells present in the combined vicinity of these two reporting centers contains double the cells contained in their individual vicinity. Therefore it can be said that clustering these two cells is not cost effective.

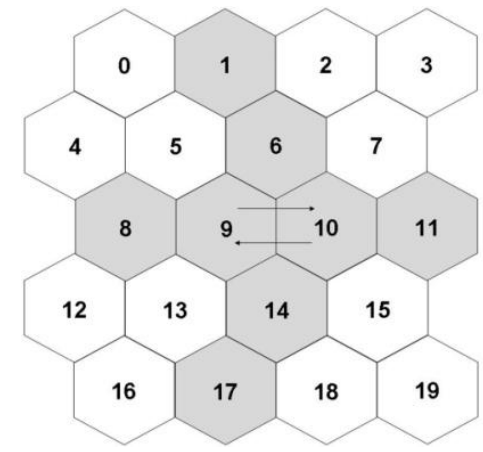

Fig 2: MT moving between adjacent reporting centers

To avoid above situation, there must be some criterion to conclude which reporting cells should be clustered and which should not. According to the proposed clustering criterion only those reporting centers that have same vicinity, should be clustered. As we can see in Fig. 2 the reporting centers 1 and 6 have same vicinity so they should be clustered. The vicinity of this cluster will include cell $0,1,2,3,4,5,6$ and 7, which is only one more than the vicinity of each individual cluster member. Similarly reporting cell 8 and 9,10 and 11, 14 and 17 can be clustered. This will increase the paging cost for MTs in these clusters only by single unit.

For bigger networks, there may be number of adjacent reporting centers with same vicinity. In such case, clustering may result into huge vicinity of the resultant cluster, which in turn will increase paging cost notably. Therefore we need a scheme that can be used with clustering technique to reduce the paging cost. In the next section, successive paging scheme is introduced that accomplishes this task effectively.

\section{SUCCESSIVE PAGING}

The idea behind successive paging comes from the fact that in bounded non reporting cell configuration, the vicinity of clusters can be divided into two or more groups of cells separated by a barrier of reporting cells as shown in Fig.3. Vicinity of clustered reporting centers 1 and 6 can be separated into two different groups consisting of cells $0,4,5$ and cells 2, 3, 7 shown by dashed lines, the cluster members (cells 1 and 6) form a barrier that separate these groups. According to proposed scheme, instead of paging whole vicinity in once it should be done in following two steps:

a) Page all the cells in any one group and all cluster members simultaneously, if target MT is found then stop.

b) Otherwise page all cells in the other group simultaneously

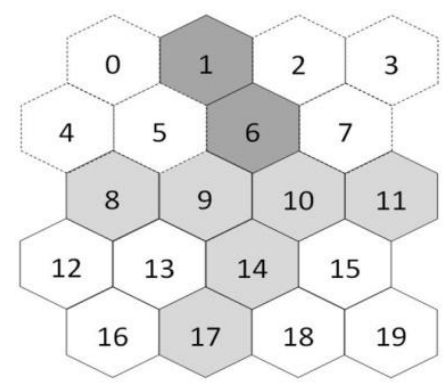

Fig 3: Clustered cell 1 and 6 with vicinity separated into two groups (dashed cells represent grouped vicinity)

For instance, if target MT (which is to be paged) is in the vicinity of cluster made up of cells 1 and 6 then fiirstly cells 0 , $4,5,1$ and 6 will be paged simultaneously and if MT is not found in these cells then cells 2, 3 and 7 will be paged 
simultaneously. Such division of paging area will reduce the probability of paging whole vicinity while locating the MT.

\section{SIMULATION AND RESULTS}

\subsection{Network Model}

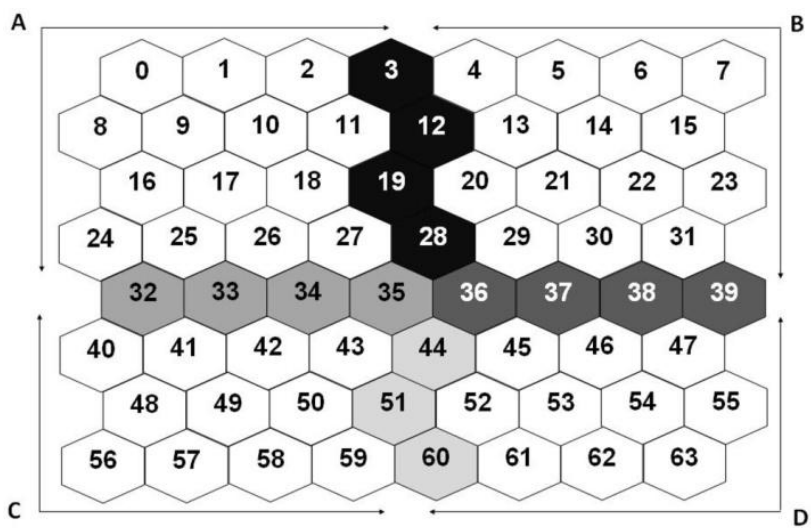

Fig 4: $8 \times 8$ Clustered network used in simulation

Network structure used in simulation is shown in Fig.4. There are 15 reporting centers that are divided into 4 clusters i.e. $3-$ 12-19-28, 32-33-34-35, 36-37-38-39 and 44-51-60. Every cluster is shown with different level of darkness. The network is divided into 4 different regions namely A, B, C and D. Vicinity of each cluster is divided into 2 groups. For instance, vicinity of Cluster 3-12-19-28 consists of region-A (14 cells) and region-B (14 cells). Similarly vicinity of clusters 32-33$34-35,36-37-38-39$ and 44-51-60 consists of 25, 24 and 21 cells. The number of MTs is set to 100 . Random walk model is used for mobility where MT has equal probability of moving into any of the six neighboring cells. Using above network structure, the performance of proposed criteria for clustering and successive paging has been evaluated by implementing the following three versions of reporting center scheme:

a) Simple Reporting Center Scheme (SRCS)

b) Clustered Reporting Center Scheme (CRCS) and

c) Clustered With Successive Paging Reporting Center Scheme (CSPS)

The SRCS is conventional reporting center scheme in which, no clustering is applied. CRCS is the resultant scheme after applying clustering in SRCS. CSPS is the resultant scheme after applying successive paging in CRCS. Each of the above schemes is simulated with 10 different values of call to mobility ratio for comparing the performance of CRCS and CSPS with SRCS in terms of LU cost, paging cost and total LM cost. Next section shows the performance comparison on the basis of results obtained from simulation.

\subsection{Location Update Cost}

The location update cost incurred by the three schemes during simulation is presented in Table.1. Since both CRCS and CSPS have used same clustering criterion, therefore both the schemes show a small variation in $\mathrm{LU}$ cost. The LU cost involved in CRCS and CSPS is much smaller as compared to SRCS, which shows that with proposed clustering criterion, the LU cost reduces up to one third as compared to the conventional reporting center scheme.
Table 1. Location update cost with varying call to mobility ratios in three schemes

\begin{tabular}{|c|c|c|c|}
\hline \multirow{2}{*}{$\begin{array}{c}\text { C/M } \\
\text { Ratio }\end{array}$} & \multicolumn{3}{|c|}{ LU Cost } \\
\cline { 2 - 4 } & SRCS & CRCS & CSPS \\
\hline 1.00 & 4440 & 1550 & 1480 \\
\hline 0.50 & 4440 & 1600 & 1400 \\
\hline 0.33 & 4440 & 1440 & 1470 \\
\hline 0.25 & 4440 & 1480 & 1510 \\
\hline 0.20 & 4440 & 1430 & 1520 \\
\hline 0.17 & 4440 & 1490 & 1440 \\
\hline 0.14 & 4440 & 1430 & 1420 \\
\hline 0.13 & 4440 & 1360 & 1380 \\
\hline 0.11 & 4440 & 1400 & 1490 \\
\hline 0.10 & 4440 & 1390 & 1480 \\
\hline
\end{tabular}

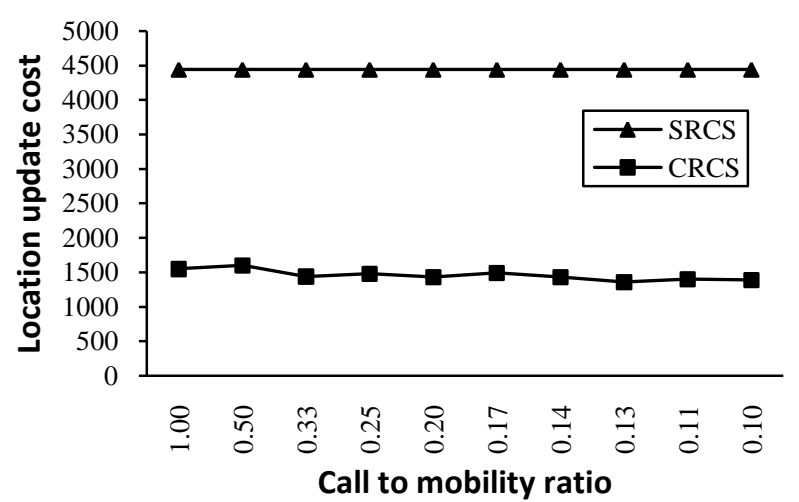

(a) SRCS vs. CRCS

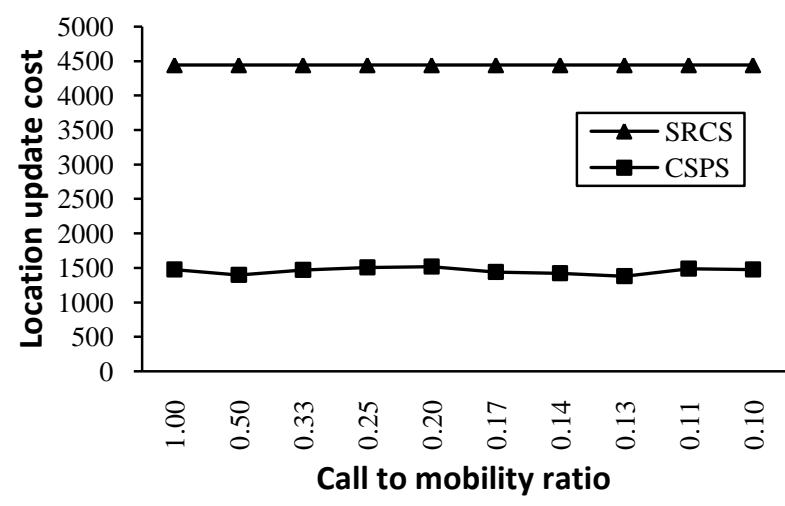

(b) SRCS vs. CSPS

Fig 5: Comparison of Location update cost

Fig.5(a) shows graph for LU cost comparison between SRCS and CRCS, Fig.5(b) shows the same between SRCS and CSPS. For simulation purpose, call to mobility ratio is varied by only varying the call arrival rates whereas mobility is kept stable therefore the LU cost is nearly stable in both the graphs. Fig.5 depicts that the location update cost involved in both CRCS and CSPS are almost same and is much less as compared to that involved in SRCS.

\subsection{Paging Cost Comparison}

Paging cost incurred by the three schemes during simulation is presented in Table.2. As stated earlier, since clustering increases paging cost therefore paging cost in CRCS is higher than SRCS. CSPS uses successive paging scheme and shows significant reduction in paging cost as shown in Table.2. 
Table.2. Paging cost with varying call to mobility ratios in three schemes

\begin{tabular}{|c|c|c|c|}
\hline \multirow{2}{*}{$\begin{array}{c}\text { C/M } \\
\text { Ratio }\end{array}$} & \multicolumn{3}{|c|}{ Paging Cost } \\
\cline { 2 - 4 } $\mathbf{1 . 0 0}$ & SRCS & CRCS & CSPS \\
\hline $\mathbf{0 . 5 0}$ & 24156 & 53867 & 31520 \\
\hline $\mathbf{0 . 3 3}$ & 14967 & 26424 & 15956 \\
\hline $\mathbf{0 . 2 5}$ & 11651 & 12764 & 9952 \\
\hline $\mathbf{0 . 2 0}$ & 9050 & 10000 & 5510 \\
\hline $\mathbf{0 . 1 7}$ & 7301 & 7995 & 4617 \\
\hline $\mathbf{0 . 1 4}$ & 5154 & 5717 & 3226 \\
\hline $\mathbf{0 . 1 3}$ & 5114 & 5606 & 3162 \\
\hline $\mathbf{0 . 1 1}$ & 4688 & 5168 & 2932 \\
\hline $\mathbf{0 . 1 0}$ & 3941 & 4329 & 2395 \\
\hline
\end{tabular}

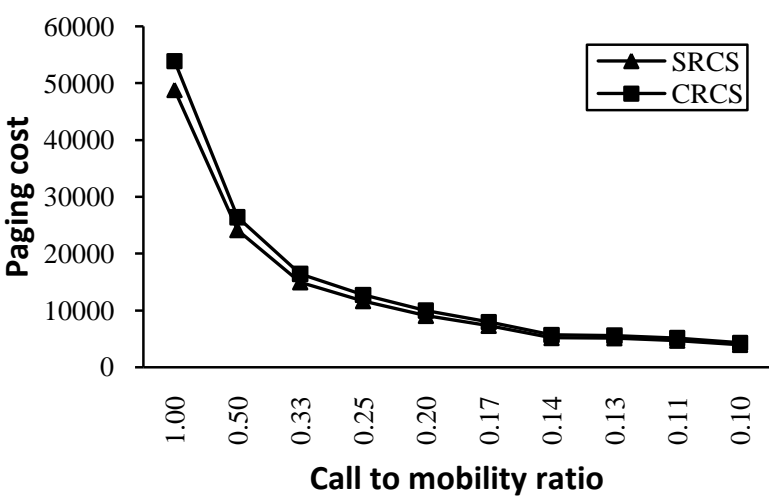

(a) SRCS vs. CRCS

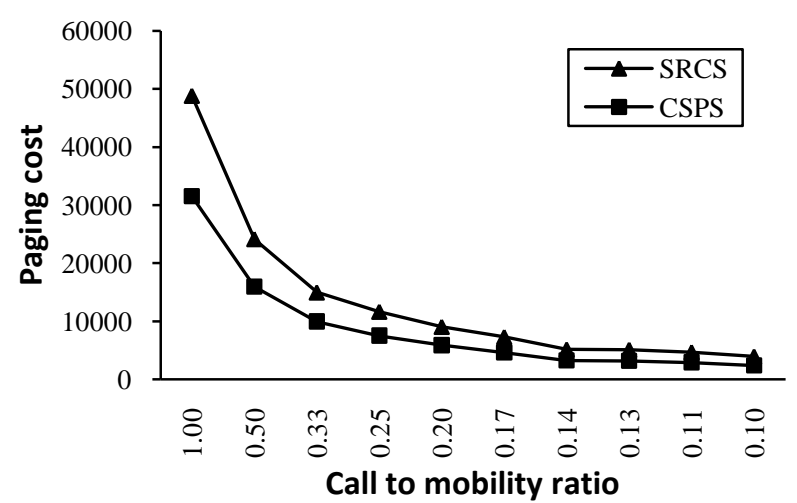

(b) SRCS vs. CSPS

Fig 6: Comparison of Paging cost

Fig.6(a) shows graph for paging cost comparison between SRCS and CRCS, whereas Fig.6(b) shows the same between SRCS and CSPS. By observing Fig.5(b) and Fig.6(b), it can be concluded that CSPS has reduced both the LU cost and paging cost significantly as compared to SRCS. As call to mobility ratio approaches smaller values, difference between paging costs decreases in both the graphs, it is due to the fall in call arrival rate, which reduces with call to mobility ratio.

\subsection{Location Management Cost}

Table. 3 shows that although considerable fall in location management cost was achieved with both the CRCS and CSPS as compared to SRCS, but CRCS showed reduction in LM cost only with smaller values of call to mobility ratio whereas CSPS had shown significant reduction in both LU cost and paging cost independent of call to mobility ratio.
Therefore CSPS outperforms both SRCS and CRCS in terms of total location management cost.

Table.3. Total location management cost with varying call to mobility ratios in three schemes

\begin{tabular}{|c|c|c|c|}
\hline \multirow{2}{*}{$\begin{array}{c}\text { C/M } \\
\text { Ratio }\end{array}$} & \multicolumn{3}{|c|}{ LM Cost } \\
\cline { 2 - 4 } $\mathbf{1 . 0 0}$ & SRCS & CRCS & CSPS \\
\hline $\mathbf{0 . 5 0}$ & 28596 & 55417 & 33000 \\
\hline $\mathbf{0 . 3 3}$ & 19407 & 28024 & 17356 \\
\hline $\mathbf{0 . 2 5}$ & 16091 & 14244 & 91422 \\
\hline $\mathbf{0 . 2 0}$ & 13490 & 11430 & 7396 \\
\hline $\mathbf{0 . 1 7}$ & 11741 & 9485 & 6057 \\
\hline $\mathbf{0 . 1 4}$ & 9594 & 7147 & 4646 \\
\hline $\mathbf{0 . 1 3}$ & 9554 & 6966 & 4542 \\
\hline $\mathbf{0 . 1 1}$ & 9128 & 6568 & 4422 \\
\hline $\mathbf{0 . 1 0}$ & 8381 & 5719 & 3875 \\
\hline
\end{tabular}

Fig.7(a) shows graph for LM cost comparison between SRCS and CRCS, Fig.7(b) shows the same between SRCS and CSPS. Fig. 8 shows comparison of total LM cost between all the three schemes. As compared to SRCS, CRCS reduces the average LM cost by $9.13 \%$ whereas CSPS reduces it by $43.24 \%$.

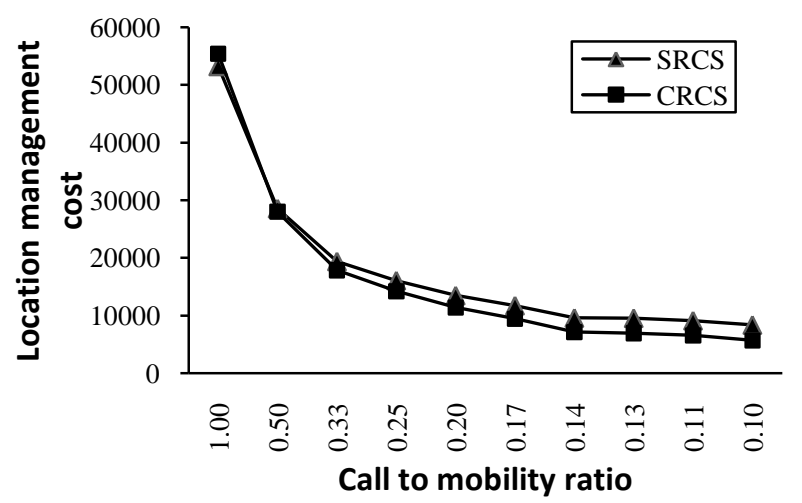

(a) SRCS vs. CRCS

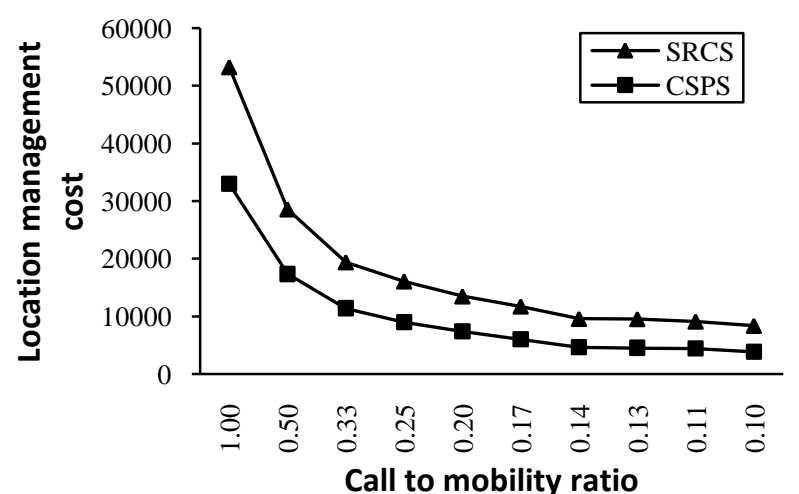

(b) SRCS vs. CSPS

Fig 7: Comparison of Total Location Management cost 


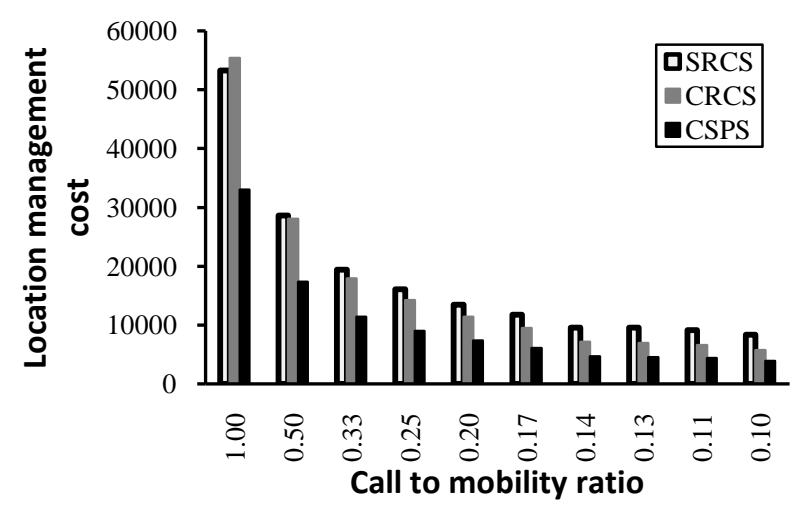

Fig 8: Comparison between location management cost of three schemes

\section{CONCLUSION}

This paper presented a simple and effective clustering criterion for bounded non reporting cell configuration of reporting center scheme in cellular mobile networks. Simulation results reveal that although clustering is individually capable of reducing location management cost but only when call to mobility ratio is small. When this clustering technique is combined with successive paging then it reduces location update cost as well as the paging cost remarkably independent of call to mobility ratio.

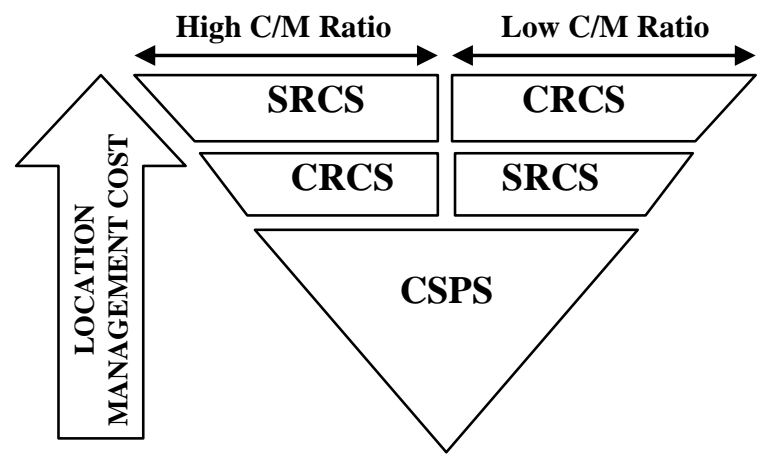

Fig 9: Location management cost involved in three schemes with respect to $\mathrm{C} / \mathrm{M}$ ratios

In Fig.9, it is shown that when call to mobility ratio is small, CRCS involves higher location management cost as compared to SRCS whereas for bigger values of call to mobility ratio, SRCS involved highest location management cost among the three schemes. But in both the cases, location management cost involved in CSPS is the smallest, which shows that CSPS outperforms both the SRCS and CRCS independent of call to mobility ratio.

\section{REFERENCES}

[1] Subrata R., and Zomaya A.Y. 2003. A Comparison of Three Artificial Life Techniques for Reporting Cell Planning in Mobile Computing. IEEE Transactions on Parallel and Distributed Systems. 142-153.
[2] Subrata R., and Zomaya A.Y. 2001. Location management in mobile computing. In Proceedings of the ACS/IEEE International Conference on Computer Systems and Applications.

[3] Gondim P.R.L. 1996. Genetic Algorithms and the Location Area Partitioning Problem in Cellular Networks. In Proceedings of the 46th IEEE Vehicular Technology Conference.

[4] Xie H., Tabbane S., and Goodman D.J. 1993. Dynamic Location Area Management and Performance Analysis. In Proceedings of the 43rd IEEE Vehicular Technology Conference Personal Comm. Freedom Through Wireless Technology.

[5] Taheri, J., and Zomaya, A.Y. 2006. Clustering Techniques for Dynamic Mobility Management. In Proceedings of the 4th ACM international workshop on Mobility management and wireless access.

[6] Mirjana I. Simić, and Predrag V. Pejović. 2009. A comparison of three methods to determine mobile station location in cellular communication systems. European Transactions on Telecommunications. 711-721.

[7] Taheri, J., and Zomaya, A.Y. 2005. A Genetic Algorithm for Finding Optimal Location Area Configurations for Mobility Management. In Proceedings of the IEEE Conference on Local Computer Networks.

[8] Taheri, J., and Zomaya, A.Y. 2005. A Simulated Annealing Approach for Mobile Location Management. In Proceedings of the 19th IEEE International Parallel and Distributed Processing Symposium.

[9] Taheri, J., and Zomaya, A.Y. 2004. The Use of a Hopfield Neural Network in Solving the Mobility Management Problem. In Proceedings of the IEEE/ACS International Conference on Pervasive Services.

[10] Subrata R., and Zomaya A.Y. 2003. Evolving Cellular Automata for Location Management in Mobile Computing Networks. IEEE Transactions on Parallel and Distributed Systems. 13-26.

[11] Biswash S. K., and Kumar C. 2011. An efficient metricbased (EM-B) location management scheme for wireless cellular networks. Journal of Network and Computer Applications. 1-16.

[12] Rodríguez Dagnino R. M., and Takagi H. 2010. Application of renewal theory to call handover counting and dynamic location management in cellular mobile networks. European Journal of Operational Research. 113.

[13] Ding Y., and Fan G. A Variation of Reporting Center Scheme. In Proceedings of the 43rd ACM Southeast Conference. 